\title{
Linking scalar elastodynamics and non-Hermitian quantum mechanics
}

\author{
Gal Shmuel ${ }^{1}$ and Nimrod Moiseyev ${ }^{2,3}$ \\ ${ }^{1}$ Faculty of Mechanical Engineering, Technion-Israel Institute of Technology, Haifa 32000, Israel \\ ${ }^{2}$ Faculty of Chemistry, Technion-Israel Institute of Technology, Haifa 32000, Israel and \\ ${ }^{3}$ Faculty of Physics, Technion-Israel Institute of Technology, Haifa 32000, Israel
}

\begin{abstract}
Recent years have seen a fascinating pollination of ideas from quantum theories to elastodynamics-a theory that phenomenologically describes the time-dependent macroscopic response of materials. Here, we open route to transfer additional tools from non-Hermitian quantum mechanics. We begin by identifying the differences and similarities between the one-dimensional elastodynamics equation and the time-independent Schrödinger equation, and finding the condition under which the two are equivalent. Subsequently, we demonstrate the application of the non-Hermitian perturbation theory to determine the response of elastic systems; calculation of leaky modes and energy decay rate in heterogenous solids with open boundaries using a quantum mechanics approach; and construction of degeneracies in the spectrum of these assemblies. The latter result is of technological importance, as it introduces an approach to harness extraordinary wave phenomena associated with non-Hermitian degeneracies for practical devices, by designing them in simple elastic systems. As an example of such application, we demonstrate how an assembly of elastic slabs that is designed with two degenerate shear states according to our scheme, can be used for mass sensing with enhanced sensitivity by exploiting the unique topology near the exceptional point of degeneracy.
\end{abstract}

\section{INTRODUCTION}

The physics of matter at the subatomic level is described by quantum mechanics. The computational complexity associated with the theory at the macroscopic scale renders it infeasible to describe the observable mechanics of materials, and hence continuum mechanics is used [1]. Despite the huge difference in the length scale that the two theories were developed for, fascinating realizations of quantum phenomena were demonstrated using macroscopic systems in recent years [2]. Examples include the Hall effect [3, 4], geometric phase [5], and negative refraction [6-10]. Special attention is given to extraordinary transport properties based on $\mathscr{P} \mathscr{T}$ symmetry [11-18], which corresponds to the commutativity of an operator with combined parity-time reversal operators. This concept originated form the discovery in quantum mechanics that Hamiltonians exhibiting this symmetry can have real eigenvalues, even if they are not Hermitian [19]. One the advantages of Non-Hermitian Quantum Mechanics (NHQM) is its quantification of the conditions for the existence of $\mathscr{P} \mathscr{T}$ symmetric Hamiltonians with a real spectrum [20].

The source of these analogies originates from the connection between the governing equations in the different branches of physics. The analogy between the time-independent Schrödinger equation and the scalar elastodynamic equation that appears in part of the literature identifies the transformation

$$
\hat{V}(x)-E \rightarrow \frac{\omega^{2}}{c^{2}(x)}
$$

where $\hat{V}(x)$ is the potential in the quantum Hamiltonian and $E$ is the energy, and in the elastic counterpart $\omega$ and $c(x)$ are the wave frequency and velocity, respectively. However, this analogy is flawed, since it mixes the operator and its eigenvalues. Using a simple transformation, we here first identify the term that appears in the one-dimensional elastodynamics equation and absent from the time-independent Schrödinger equation. In turn, this derivation allows us to determine the condition under which the two equations are equivalent. Following this analysis, we apply tools from the non-Hermitian formalism of quantum mechanics to elastodynamics, in addition to those transferred recently [21, 22], as described next.

First, we show the application of the time-independent Rayleigh-Schrödinger perturbation theory of quantum mechanics to elastodynamics $[23,24]$. This theory provides the solutions of a perturbed Hamiltonian in terms of a series expansion about an Hermitian Hamiltonian, where its nonHermitian formalism determines the radius of convergence by extending the perturbation to the complex plane. By way of example, we consider an elastic assembly composed of a PMMA slab that is perfectly bonded between two steel slabs which are fixed at the ends. We apply the aforementioned theory to calculate the shear response of an elastic assembly whose properties are complex perturbations of the original assembly. This response is given in terms of a perturbation expansion, for which we calculate its radius of convergence [25]. Importantly, in this process we also calculate the exceptional point (EP) - the point at which the spectrum of the perturbed assembly has a non-Hermitian degeneracy, where two of its eigenmodes coalesce, together with their corresponding complex frequencies [26-28]. This occurs in our example for an assembly comprising a lossy slab with specific viscoelastic shear modulus [29].

Subsequently, we present a proof of concept how this assembly, i.e., an elastic assembly with non-Hermitian degeneracy in its spectrum, can be utilized for mass sensing with enhanced sensitivity. Specifically, by a combination of algebraic arguments and numerical calculations, we show that when a mass is deposited, the degenerate frequency of the elastic assembly splits into two frequencies, such that the splitting is proportional to the square root of the mass. This phenomenon is the physical manifestation of the topology near an EP in the spectrum of our elastodynamic problem. Accordingly, measurement of the frequency splitting quantifies the weight of the deposited mass, with higher mass responsivity at small masses. By contrast, standard mechanical sensors measure 
the shift in the mechanical resonant frequency, which scales linearly with the deposited mass, hence of inferior sensitivity at small masses $[30,31]$. Indeed, the square-root topology near an EP has been used in other systems for sensing [32, 33], most recently by Djorwe et al. [34] using optomechanical cavities coupled by mechanical resonators.

The second analogy we draw is between the NHQM formalism of the particle in a box model and the previous elastic assembly, when the steel slabs are semi-infinite. This problem corresponds to a non-Hermitian Hamiltonian, owing to (radiation) outgoing boundary conditions. Only in the nonHermitian formalism of quantum mechanics the poles of the scattering matrix are associated with metastable states, where the imaginary part of the poles provides the resonance width, or rate of decay of resonance state [35]. Here, we obtain the physical counterparts of these quantities in the elastodynamic settings. Specifically, we show that the imaginary part of the poles of the elastic scattering matrix equals half the decay rate of the mechanical energy in the PMMA slab, associated with leaky modes in the elastic assembly.

Finally, we develop a real perturbation theory for the nonHermitian system using the NHQM complex scaling method $[36,37]$. With the framework developed in this Section, we are able to derive the eigenstates of the perturbed Hamiltonian as an expansion about a non-Hermitian Hamiltonian with real parameters, such as the stiffness and length of a fourth slab in our example. Thereby, we constitute a framework to analyze and construct degeneracies by real perturbations, although its numerical study is beyond our scope here [38]. It has already been established that systems exhibit extraordinary behavior in the vicinity of non-Hermitian degeneracies, such as ultra-sensitivity [39] (as we also demonstrate in the sequel), Berry phase acquiring [40], and asymmetric scattering properties $[41,42]$. Accordingly, our framework offers an approach to achieve such extraordinary wave phenomena by designing non-Hermitian degeneracies in simple elastic systems, without the need for external gain and loss as in the works mentioned earlier.

The results described above are presented in the following order. Section II provides a short summary of the elastodynamics equations, and specifically their reduced form in the scalar (one-dimensional) setting. Section III identifies the similarities and differences between the scalar elastodynamic equation and the time-independent Schrödinger equation, based on a transformation we develop. Section IV formulates the elastic counterpart of the NHQM timeindependent Rayleigh-Schrödinger theory, demonstrates the calculation of the radius of convergence for our model slabs problem, and importantly determines the EP in the spectrum of the slabs. Section V demonstrates how the elastic assembly that exhibits a non-Hermitian degeneracy can be utilized for mass sensing with enhanced sensitivity. Section VI details the calculation of metastable states and energy decay when the steel slabs are semi-infinite, using the analogy with the NHQM particle in a box model. The development of a real perturbation theory for non-Hermitian elastic systems is carried out in Section VII. A summary of our results and outlook concludes the paper in Section VIII.

\section{THE ELASTODYNAMICS EQUATIONS IN CONTINUUM MECHANICS}

The continuum governing equations are based on the hypothesis that the inter-particle forces can be replaced by the stress tensor field $\boldsymbol{\sigma}$; in terms of $\boldsymbol{\sigma}$, the balance of linear momentum yields [43]

$$
\nabla \cdot \boldsymbol{\sigma}(\mathbf{x}, t)=\rho(\mathbf{x}, t) \ddot{\mathbf{u}}(\mathbf{x}, t),
$$

where $\rho$ is the mass density and $\mathbf{u}$ is the displacement vector field of material points. The stress is related to the displacement field via the constitutive equation

$$
\boldsymbol{\sigma}=\mathbf{C}(\mathbf{x}) \nabla \mathbf{u},
$$

where $\mathbf{C}$ is the fourth-order elasticity tensor. If the material is locally isotropic, the tensor $\mathbf{C}$ is a function of the Lame parameters $\mu(\mathbf{x})$ and $\lambda(\mathbf{x})$, and the combination of Eqs. (1)(2) can be put in the form

$$
\{\nabla[\lambda(\mathbf{x}) \nabla \cdot+2 \mu(\mathbf{x}) \nabla \cdot]-\nabla \times[\mu(\mathbf{x}) \nabla \times]\} \mathbf{u}=\rho(\mathbf{x}) \ddot{\mathbf{u}} .
$$

Eq. (3) exposes the unique coupling in elastodynamics between the volumetric part of the vector field, proportional to $\nabla \cdot \mathbf{u}$, and its transverse or shear part, proportional to $\nabla \times \mathbf{u}$. This coupling has a significant effect on the Hermiticity of the system, discussed elsewhere [10]. When considering one-dimensional motions, the coupling is eliminated and the problem reduces to a scalar one. Using the ansatz $u(x, t)=U(x) e^{-i \omega t}$, Eq. (3) then reduces to

$$
-\frac{\mathrm{d}}{\mathrm{d} x} \tilde{\mu} \frac{\mathrm{d}}{\mathrm{d} x} U(x)=\rho(x) \omega^{2} U(x),
$$

where $\tilde{\mu}=\mu$ when the displacements are normal to the $x$ direction (termed transverse or shear waves), and $\tilde{\mu}=\lambda+2 \mu$ when the displacements are along the $x$ direction (termed pressure or volumetric waves). In what follows we focus on the former, bearing in mind that the same analysis holds for the latter, by carrying out a change of modulus.

\section{SIMILARITIES AND DIFFERENCES BETWEEN THE 1D ELASTODYNAMIC EQUATION AND THE TIME-INDEPENDENT SCHRÖDINGER EQUATION}

The objective of this Section is to transform the equation of elastodynamics in the one-dimensional case to a Schrödingertype equation, in order to highlight the similarities and differences between them. To this end, we first multiply Eq. (4) by $\mu^{-1}(x)$, and define $c^{2}(x)=\mu(x) / \rho(x)$ to obtain

$$
-\frac{1}{\mu} \frac{\mathrm{d}}{\mathrm{d} x} \mu \frac{\mathrm{d}}{\mathrm{d} x} U=\frac{\omega^{2}}{c^{2}} U .
$$

Observe that in terms of the variable $\eta(x)=\mu^{-1}(x)$, the lefthand side equals

$$
-\eta \frac{\mathrm{d}}{\mathrm{d} x} \frac{1}{\eta} \frac{\mathrm{d}}{\mathrm{d} x} U=\left[\frac{1}{\eta} \frac{\mathrm{d} \eta}{\mathrm{d} x} \frac{\mathrm{d}}{\mathrm{d} x}-\frac{\mathrm{d}^{2}}{\mathrm{~d} x^{2}}\right] U
$$




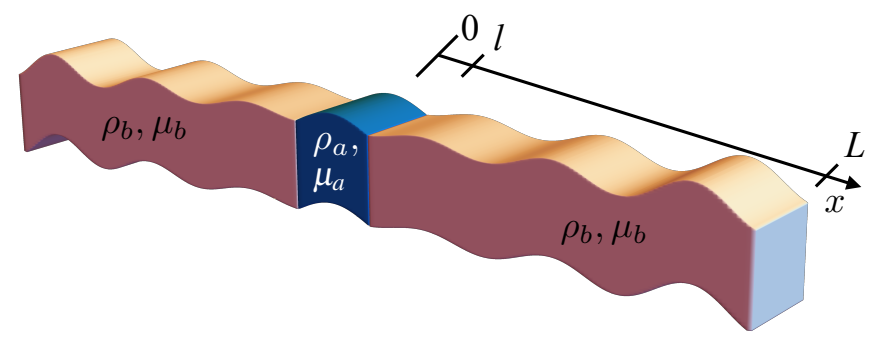

FIG. 1. An assembly of three elastic slabs. The Hermitian case corresponds to fixed boundaries at $x= \pm L$, and the non-Hermitian case corresponds to an infinite assembly with outgoing boundary conditions.

By further defining $n^{2}(x)=\eta(x)$, we rewrite Eq. (5) as

$$
\left[2 \frac{1}{n} \frac{\mathrm{d} n}{\mathrm{~d} x} \frac{\mathrm{d}}{\mathrm{d} x}-\frac{\mathrm{d}^{2}}{\mathrm{~d} x^{2}}\right] U=\frac{\omega^{2}}{c^{2}} U
$$

Finally, we employ the transformation $U(x)=n(x) \Psi(x)$ and multiply Eq. (7) by $c^{2} / n$ to achieve the form

$$
\hat{H} \Psi(x)=\omega^{2} \Psi(x),
$$

where $\omega^{2}$ is the eigenvalue, and $\hat{H}=\hat{T}+\hat{V}_{C M}$ with

$$
\hat{T}=-c^{2}(x) \frac{\mathrm{d}^{2}}{\mathrm{~d} x^{2}}, \quad \hat{V}_{C M}=2\left(\frac{c(x)}{n(x)} \frac{\mathrm{d} n}{\mathrm{~d} x}\right)^{2}-\frac{c^{2}(x)}{n(x)} \frac{\mathrm{d}^{2} n}{\mathrm{~d} x^{2}}
$$

The operator $\hat{V}_{C M}$, which is a local function of $x$ and does not involve spatial derivatives, can be interpreted as the potential of a conservative force. By further separating $\hat{T}$ according to

$$
\hat{T}=\hat{T}_{Q M}+\hat{T}_{C M}, \hat{T}_{Q M}=-\frac{\mathrm{d}}{\mathrm{d} x} c^{2}(x) \frac{\mathrm{d}}{\mathrm{d} x}, \hat{T}_{C M}=\frac{\mathrm{d} c^{2}}{\mathrm{~d} x} \frac{\mathrm{d}}{\mathrm{d} x},
$$

we can identify $\hat{T}_{Q M}$ with the kinetic energy operator in the Schrödinger equation of an electron with an effective mass

$$
m_{\mathrm{eff}}(x)=\frac{1}{2 c^{2}(x)},
$$

that varies when the electron traverses different semiconductors. The difference between the equations thus amounts to $\hat{T}_{C M}$ - this term does not have the form of a kinetic energy operator nor a potential, as it involves one spatial derivative.

To draw the analogy with the NHQM model problem of a particle in a box with outgoing boundary conditions, we consider the prevalent case of a solid that is composed of different homogeneous slabs. The medium properties are therefore piecewise constant. For simplicity, we consider two constituents, say, material $a$ with $\rho_{a}$ and $\mu_{a}$, which is perfectly bonded at $x= \pm l$ to two infinite slabs made of a stiffer material $b$ with $\rho_{b}$ and $\mu_{b}>\mu_{a}$ (Fig. 1).

If we further assume that

$$
\frac{\mu_{a}}{\rho_{a}}=\frac{\mu_{b}}{\rho_{b}}
$$

then $\hat{T}_{C M}$ vanishes; in this case-and this case only-there is an exact analogy between the one-dimensional equation of elastodynamics and the time-independent Schrödinger equation. The corresponding potential $\hat{V}_{C M}$ exhibits a potential well between two infinite barriers (spikes), owing to the jump discontinuities of $\mu(x)$, and hence of $n(x)$. In the equivalent quantum particle in a box model, there are resonance phenomena and metastable states associated with complex eigenvalues that are elegantly analyzed and explained using the NHQM formalism [35]. In the sequel, we will show that this formalism establishes a powerful machinery to study corresponding elastodynamic phenomena, even when restriction (12) is removed and the exact analogy is broken. Before we proceed, it is useful to note that for two-dimensional elastodynamics, by contrast, an exact analogy with the timeindependent Schrödinger equation always exists. To show this, it is sufficient to consider anti-plane shear waves of the form

$$
u(x, y, t)=Y(y) e^{i\left(k_{x} x-\omega t\right)}
$$

propagating in a medium that is laminated in the $y$ direction. In each lamina, Eq. (3) reduces to the following equation for $Y(y)$

$$
\left[\frac{\mathrm{d}^{2}}{\mathrm{~d} y^{2}}+\frac{\omega^{2}}{c^{2}(y)}\right] Y(y)=k_{x}^{2} Y(y)
$$

In this case, it is possible to identify $k_{x}^{2}$ (not $\omega^{2}$ ) as the eigenvalue to be determined, $\mathrm{d}^{2} / \mathrm{d} y^{2}$ with the kinetic energy operator, and $\omega^{2} / c^{2}(y)$ with the potential. Physically, Eq. (14) represents the question: given excitation frequency and mechanical properties, what would be the propagation constant in the direction perpendicular to the material modulation? $c f$. Ref. [44] on a similar analogy between the timeindependent Schrödinger equation and Maxwell equations.

Returning to the one-dimensional problem, we consider next the transformation $U(x)=\rho^{-1 / 2}(x) \Psi(x)$, which upon substitution into Eq. (4) and its multiplication by $\rho^{-1 / 2}(x)$ provides a different representation of the Hamiltonian, namely,

$$
\hat{H}=-\frac{1}{\sqrt{\rho(x)}} \frac{\mathrm{d}}{\mathrm{d} x} \mu(x) \frac{\mathrm{d}}{\mathrm{d} x} \frac{1}{\sqrt{\rho(x)}} .
$$

For real moduli, this Hamiltonian is Hermitian if it operates on functions that vanish at the boundary of the problem domain, and therefore the eigenfunctions $\{\Psi(x)\}$ are orthogonal one to each other; the application of NHQM perturbation theory to such Hermitian systems in 1D elastodynamics is demonstrated first.

\section{NHQM PERTURBATION THEORY FOR ELASTODYNAMICS: THE MODEL PROBLEM OF A FINITE SLAB}

In quantum mechanics, the standard time-independent Rayleigh-Schrödinger theory provides the solutions of a perturbed Hamiltonian in terms of a series expansion about an Hermitian Hamiltonian. The NHQM formalism determines 
the radius of convergence by extending the perturbation to the complex plane and calculating the EP-the point at which the perturbed Hamiltonian has a non-Hermitian degeneracy $[27,28,35]$. The process is exemplified in this Section, by calculating first the eigenfrequencies and eigenmodes of an Hermitian Hamiltonian that models an elastodynamic system made of purely elastic and finite slabs; subsequently, we determine the convergence radius of the elastodynamic RayleighSchrödinger expansion by calculating the EP in the perturbed non-Hermitian Hamiltonian spectrum.

Thus, we truncate the assembly at $x= \pm L$, and fix the boundaries such that the displacement field vanishes at the edges. The standard procedure to calculate the real frequencies starts with the ansatz

$$
U^{(0)}(x)= \begin{cases}A \cos k_{a} x+B \sin k_{a} x, & x<|l|, \\ C_{+} \sin k_{b}(x-L), & l<x<L, \\ C_{-} \sin k_{b}(x+L), & -L<x<-l,\end{cases}
$$

where owing to Eq. (3) and the continuity of $u(x, t)$

$$
\omega^{2}=c_{i}^{2} k_{i}^{2}, \quad i=a, b,
$$

and hence $k_{a}$ and $k_{b}$ are related via

$$
\frac{k_{a}}{k_{b}}=\frac{c_{b}}{c_{a}} .
$$

The continuity of the spatial parts of the displacement and stress at $x= \pm l$ takes the form

$$
\begin{aligned}
A \cos k_{a} l & =C \sin k_{b}(l-L), \\
-A \mu_{a} k_{a} \sin k_{a} l & =\mu_{b} k_{b} C \cos k_{b}(l-L),
\end{aligned}
$$

from which the relation between the amplitudes $A$ and $C$ is determined. The resultant transcendental equation for the eigenfrequencies is

$$
\begin{aligned}
\tan \frac{\omega l}{c_{a}} & =\gamma \tan \frac{\omega(l-L)}{c_{b}}, & & \text { (odd modes) } \\
\cot \frac{\omega l}{c_{a}} & =-\gamma \tan \frac{\omega(l-L)}{c_{b}}, & & \text { (even modes). }
\end{aligned}
$$

We denote the eigenfrequencies by $\left\{\omega_{m}^{(0)}\right\}_{m \in \mathbb{N}}$, and the corresponding transformed eigenfunctions of Eq. (15) by $\Psi_{m}^{(0)}(x)$. It is clear that $\left\{\omega_{m}^{(0)}\right\}_{m \in \mathbb{N}}$ are real and the Hamiltonian is indeed Hermitian.

Consider next another assembly, obtained by replacing the right half of the central slab by a slab whose shear stiffness is $\alpha$. The resultant Hamiltonian can be written as a sum of the Hamiltonian of the original medium, denoted $\hat{H}^{(0)}$, and a perturbation $\alpha \hat{H}^{(1)}$, where

$$
\hat{H}^{(1)}=-\frac{1}{\sqrt{\rho_{a}}} \frac{\mathrm{d}}{\mathrm{d} x} \frac{\mathrm{d}}{\mathrm{d} x} \frac{1}{\sqrt{\rho_{a}}}
$$

operates on functions over $0<x<l$. Up to a critical value of $\alpha$, NHQM perturbation theory can deliver the response of the perturbed assembly, in terms of $\Psi_{m}^{(0)}(x)$ as the zero-order solutions [45]. Using the standard time-independent RayleighSchrödinger theory, we obtain the $n^{\text {th }}$ order correction terms $\omega^{(j)}$ and $\Psi_{m}^{(j)}(x)$, namely,

$$
\omega_{m}^{2}(x ; \alpha)=\sum_{j=0}^{\infty} \alpha^{j} \omega_{m}^{2(j)}, \quad \Psi_{m}(x ; \alpha)=\sum_{j=0}^{\infty} \alpha^{j} \Psi_{m}^{(j)} ;
$$

the convergence of these sums is limited to values of $\alpha-$ including complex values-inside a circle in the complex plane whose origin is 0 and its radius is denoted $\left|\alpha^{E P}\right|$. This radius equals the radius of the complex branch point at which two adjacent modes coalesce. Thus, a non-Hermitian degeneracy is obtained when the conditions

$$
U_{m}\left(\alpha^{E P}\right)=U_{m \pm 1}\left(\alpha^{E P}\right) \equiv U_{m}^{E P}
$$

and

$$
\Psi_{m}\left(x ; \alpha^{E P}\right)=\Psi_{m \pm 1}\left(x ; \alpha^{E P}\right) \equiv \Psi_{m}^{E P}(x)
$$

are satisfied. Since for any value of $\alpha \neq \alpha^{E P}$ the two modes $\Psi_{m}\left(x ; \alpha^{E P}\right)$ and $\Psi_{m \pm 1}\left(x ; \alpha^{E P}\right)$ are orthogonal one to another, at the EP $\Psi_{m}^{E P}(x)$ is self-orthogonal, as the two solutions coalesce [35]. To determine $\alpha^{E P}$, we first represent $\hat{H}^{(0)}$ and $\hat{H}^{(1)}$ using the matrices $\mathrm{H}^{(0)}$ and $\mathrm{H}^{(1)}$, defined by

$$
\mathrm{H}^{(\alpha)}{ }_{m n}=\int_{\mathscr{I}} \hat{\Psi}_{m}^{(0)} \hat{H}^{(i)} \hat{\Psi}_{n}^{(0)} \mathrm{d} x, \quad i=0,1,
$$

where $\mathscr{I}=[-L, L]$ and $[0, l]$ when $i=0$ and 1 , respectively, and

$$
\hat{\Psi}_{m}^{(0)}=\Psi_{m}^{(0)} / \int_{-L}^{L} \Psi_{m}^{2(0)} \mathrm{d} x .
$$

Note that the standard procedure to derive orthogonality relations for real functions provides

$$
\left\langle\hat{\Psi}_{m}^{(0)}, \hat{\Psi}_{n}^{(0)}\right\rangle:=\int_{-L}^{L} \hat{\Psi}_{m}^{(0)} \hat{\Psi}_{n}^{(0)} \mathrm{d} x=\delta_{m n} .
$$

We are now at the position to seek the smallest $\alpha$ for which the matrix

$$
\mathrm{H}(\alpha)=\mathrm{H}^{(0)}+\alpha \mathrm{H}^{(1)}
$$

has an eigenvalue multiplicity, using a modified Newton's method [46]. To proceed with numerical computations, we consider by way of example a middle slab made of PMMA, which is bonded between two steel slabs, whose properties are

$$
\begin{array}{ll}
\rho_{a}=1200 \mathrm{~kg} \mathrm{~m}^{-3}, & \mu_{a}=1.21 \mathrm{GPa}, \quad l=1 \mathrm{~cm} \\
\rho_{b}=7800 \mathrm{~kg} \mathrm{~m}^{-3}, & \mu_{b}=78.85 \mathrm{GPa}, \quad L=3 \mathrm{~cm} .
\end{array}
$$

For simplicity, we truncate the size of $\mathrm{H}^{(\alpha)}$ to $2 \times 2$ using the first odd and even modes.

The results are shown in Fig. 2 in a dimensionless form (lengths are divided by $L$, mass densities and shear moduli are divided by the mean value of the quantity when averaged between the two phases). 
(a)

(b)
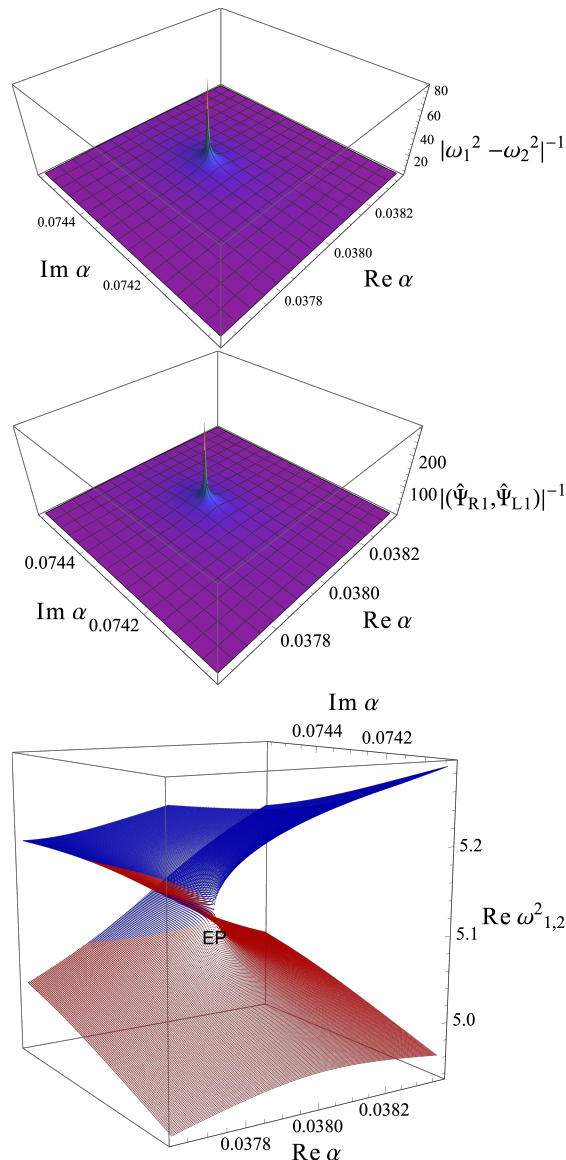

(d)

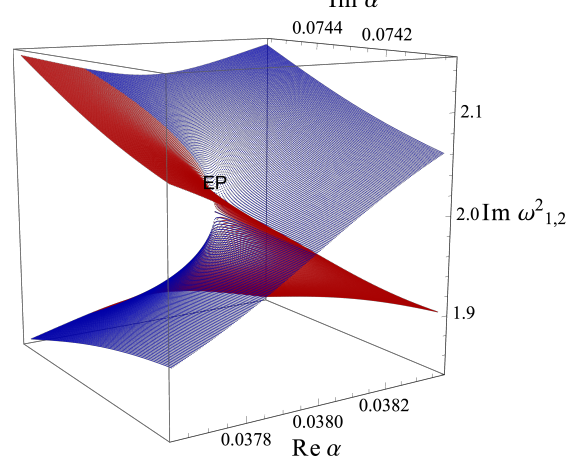

FIG. 2. (a) The inverse of the absolute value of difference between the eigenvalues as function of $\alpha$. Observe that $\lim _{\alpha \rightarrow \alpha^{E P}}\left|\omega_{1}^{2}-\omega_{2}^{2}\right|^{-1}=\infty$. (b) The inverse of the inner product between the first right and left eigenvectors as function of $\alpha$. Observe that $\lim _{\alpha \rightarrow \alpha^{E P}}\left|\left\langle\hat{\Psi}_{\mathrm{R} 1}, \hat{\Psi}_{\mathrm{L} 1}\right\rangle\right|^{-1}=\infty$. (c) The real part of the eigenvalues as function of $\alpha$. The red and blue surfaces correspond to $\omega_{1}^{2}$ and $\omega_{2}^{2}$, respectively. (d) The imaginary part of the eigenvalues as function of $\alpha$. (Same color legend as in the previous panel.) At $\alpha=\alpha^{E P}$ the two eigenvalues coalesce to the complex $\omega^{E P}$.

Specifically, Fig. 2a shows the inverse of the absolute value of the difference between the two eigenvalues of $\mathrm{H}$ versus $\operatorname{Re} \alpha$ and $\operatorname{Im} \alpha$; the peak at $\alpha \simeq 0.38+0.074 i$ identifies $\alpha^{E P}$, and hence the radius of convergence. We note that such a value of $\alpha$, i.e., with a positive imaginary part, physically corresponds to a viscoelastic slab, and hence realzing this
EP does not require any gain. Fig. $2 b$ shows the inverse of the inner product between the first right $\left(\hat{\Psi}_{\mathrm{R} 1}\right)$ and left $\left(\hat{\Psi}_{\mathrm{L} 1}\right)$ eigenvectors as function of $\alpha$. Indeed, we observe that $\lim _{\alpha \rightarrow \alpha^{E P}}\left|\left\langle\hat{\Psi}_{\mathrm{R} 1}, \hat{\Psi}_{\mathrm{L} 1}\right\rangle\right|^{-1}=\infty$, confirming that this is a nonHermitian degeneracy, as that the corresponding functions are self-orthogonal [47]. Finally, we show that the spectrum in the vicinity of the EP exhibits a Riemann surface structure-the signature of non-Hermitian degeneracy-by plotting the real (Fig. 2c) and imaginary (Fig. 2d) parts of $\omega_{1}^{2}$ (red surface) and $\omega_{2}^{2}$ (blue surface). We denote for later use the frequency at the EP by $\omega^{E P}$, such that

$$
\omega^{E P}:=\omega_{1}\left(\alpha=\alpha^{E P}\right)=\omega_{2}\left(\alpha=\alpha^{E P}\right) .
$$

\section{APPLICATION TO MASS SENSING}

We demonstrate next how the unique topology near the EP can be harnessed to design a mass sensor with enhanced sensitivity, based on the aforementioned assembly when tuned to operate at the EP. First, we recall that standard mechanical mass sensors are based on the shift in the mechanical resonant frequency, owing to any deposited mass [30]. For small masses, the shift is linear in the perturbation [31, 48]. This linear relation can be interpreted as the first term in the Taylor series of the frequency as function of the mass

$$
\operatorname{Re} \omega(m)-\operatorname{Re} \omega(0)=S_{T} m+\mathscr{O}\left(m^{2}\right),
$$

where $m$ is the mass of the deposited element, $\operatorname{Re} \omega(0)$ is the (real part of the) resonant frequency of the unperturbed system, and $S_{T}=\partial \operatorname{Re} \omega(m) / \partial m$ at $m=0$. By contrast, there is not a Taylor expansion of the frequency shift from an EP, however it does admit a Puiseux Series. In case when the EP is of two eigenvalues and eigenmodes, we have that

$$
\operatorname{Re} \omega(m)-\operatorname{Re} \omega^{E P}=S_{P} \sqrt{m}+\mathscr{O}(m),
$$

with some coefficient $S_{P}$. Sensing of a device is thus quantified by the so-called the mass responsivity $R=\partial \operatorname{Re} \omega / \partial m$ $[31,49]$. It is clear that conventional sensors have a finite $R$ as $m \rightarrow 0$, whereas for EP-based sensors

$$
\lim _{m \rightarrow 0} \frac{\partial \operatorname{Re} \omega(m)}{\partial m} \propto \lim _{m \rightarrow 0} \frac{1}{\sqrt{m}}=\infty,
$$

i.e., theoretically an infinite sensitivity, which in practice is limited by the resolution of the frequency measurement. This feature has been employed for sensors in different physical systems [32, 33], and specifically in systems comprising optomechanical cavities coupled by mechanical resonators [34]. Here, we apply this approach to the elastodynamic system described in the previous section, emphasizing that its EP does not require any realization of gain, as that assembly comprises only elastic and viscoelastic slabs. This is carried out by calculating the eigenfrequencies when a discrete element with mass $m$ is deposited at the center of the assembly whose Hamiltonian is given by Eqs. (30)-(31) with 

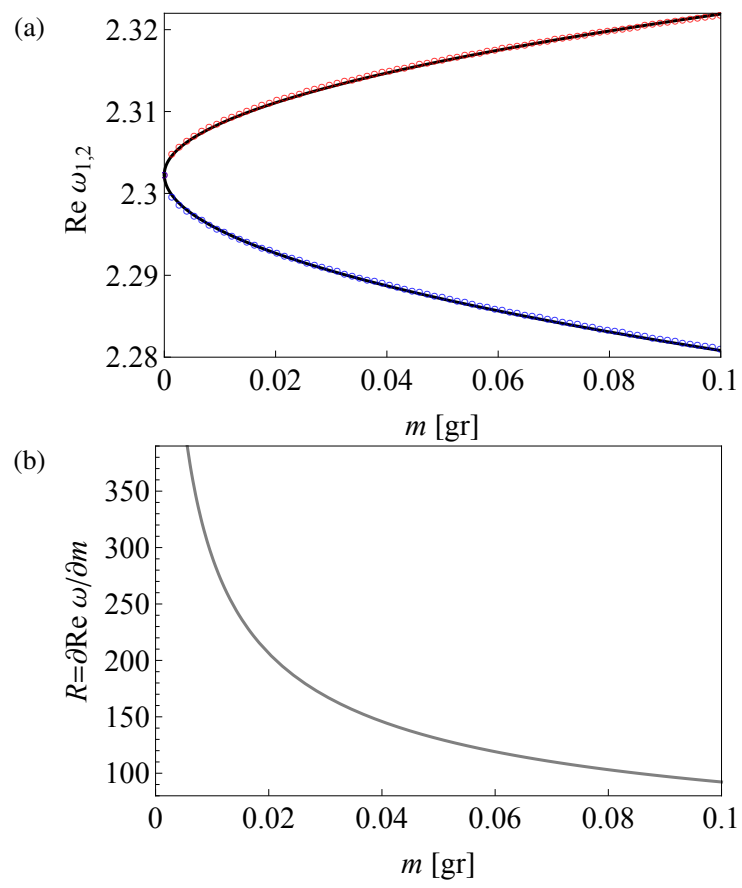

FIG. 3. (a) The real part of the first (blue circles) and second (red circles) eigenfrequencies of the our elastodynamic system, when operating at an EP and augmented by a deposited mass $m$ (in grams). The solid lines are the functions (36), which are proportional to $\sqrt{m}$, and their agreement with the calculated frequencies confirms the square-root nature of the frequency splitting. By contrast, conventional mechanical sensors are linear in $m$. (b) The mass responsivity $R=\partial \operatorname{Re} \omega / \partial m$ of device as function of $m$. Notice the theoretical infinite responsivity in the limit of an infinitesimal mass.

$\alpha=\alpha^{E P}$. The mass is modeled by replacing $\rho(x)$ with $\rho(x)+\delta \rho(x)$ in the Hamiltonian (15) where $\delta \rho(x)=\delta \rho_{0}$ over $-L / 50<x<L / 50$ such that $m=\delta \rho_{0} L / 100$ [50], and calculating the resultant eigenvalues of Eq. (30). Fig. 3a depicts (the real part of) the first (blue circles) and second (red circles) eigenfrequencies for representative values of the deposited mass (in grams). It is shown how frequency splitting occurs owing to the added mass, in a manner that is nonlinear in the perturbation-the smaller the mass, the greater the relative change. The solid lines are the functions

$$
\operatorname{Re} \omega^{E P}+S_{P} \sqrt{m}
$$

where $S_{P}=-2.02$ and 1.845 for the lower and upper curves, respectively; the matching between Eq. (36) and the calculated frequencies thereby confirms the conjectured square root nature of the frequencies dependency in the deposited mass. Again, we emphasize that the scaling is linear in conventional mechanical sensors based on the shift of the resonant frequency, therefore inferior for extremely small masses.

The enhanced sensitivity near the EP is further highlighted in Fig. 3b, by plotting the mass responsivity as function of $m$, using the derivative of the fitted function (36) for the higher frequency. Thereby, we show the theoretical infinite responsivity in the limit of an infinitesimal mass.

\section{A NON-HERMITIAN MODEL PROBLEM WITH OUTGOING BOUNDARY CONDITIONS}

We pursue next the analogy drawn in Section III between the infinite elastic assembly and the model problem in NHQM of a particle in a box with outgoing boundary conditions, thereby presenting the physical interpretation of this theory for elastodynamics. Specifically, we will demonstrate that the NHQM approach will provide the so-called leaky eigenmodes of the system, whose imaginary part of the eigenvalues delivers the decay rate of the elastic energy in the middle slab.

Accordingly, when the steel slabs now extend to $\pm \infty$ and the PMMA slab is released from some arbitrary initial shear deformation, we impose outgoing boundary conditions and seek solutions to $U(x)$ in the form

$$
U(x)= \begin{cases}A \cos k_{a} x+B \sin k_{a} x, & x<|l|, \\ C_{+} e^{i k_{b}(x-l)}, & x>l, \\ C_{-} e^{-i k_{b}(x+l)}, & x<-l .\end{cases}
$$

(The relation between $k_{a}, k_{b}$ and $\omega$ remains as in the Hermitian problem.) The continuity of the displacement and stress at $x= \pm l$ now reads

$$
\begin{aligned}
A \cos k_{a} l \pm B \sin k_{a} l & =C_{ \pm}, \\
\mu_{a} k_{a}\left(\mp A \sin k_{a} l+B \cos k_{a} l\right) & = \pm C_{ \pm} \mu_{b} i k_{b} .
\end{aligned}
$$

Manipulating these equations provides

$$
\begin{array}{rlrl}
i \tan k_{a} l & =\gamma, & & \text { (odd modes) } \\
i \cot k_{a} l & =-\gamma, & \text { (even modes) }
\end{array}
$$

where the impedance mismatch $\gamma$ is

$$
\gamma=\frac{\mu_{a} k_{a}}{\mu_{b} k_{b}}=\frac{\mu_{a} c_{b}}{\mu_{b} c_{a}}=\sqrt{\frac{\mu_{a} \rho_{a}}{\mu_{b} \rho_{b}}} .
$$

Eqs. (40)-(41) are solved by

$$
k_{a} l=-i \operatorname{arctanh} \gamma+\frac{m \pi}{2}, m \in \mathbb{Z},
$$

where odd and even $m$ correspond to even and odd modes, respectively. Hence, there are infinitely many discrete complex solutions with a different real part and the same imaginary part; there are no bound states associated with real solutions. The obtained roots are the poles of the scattering matrix, which only in the non-Hermitian formalism of quantum mechanics delivers fundamental information on the modes, without the need to carry out wave packet calculations. For example, in NHQM the imaginary part of the poles provides the resonance width, or rate of decay of resonance state [35]. Here, by analogy, the imaginary part in Eq. (43) should provide information on the decay rate of the mechanical energy. This relation is demonstrated in Fig. 4, where the log of the mechanical energy stored in the PMMA slab

$$
E_{a}(t)=\frac{1}{2} \int_{-l}^{l}\left(\mu_{a} u_{, x}^{2}+\rho_{a} u_{, t}^{2}\right) \mathrm{d} x
$$


is evaluated as function of $t$, when calculated using the finite volume method [51] for some (real) arbitrary initial conditions. Indeed, the slope of its linear interpolation (red curve) matches $-2 \operatorname{Im} \omega$, and is independent of the form of the initial conditions.

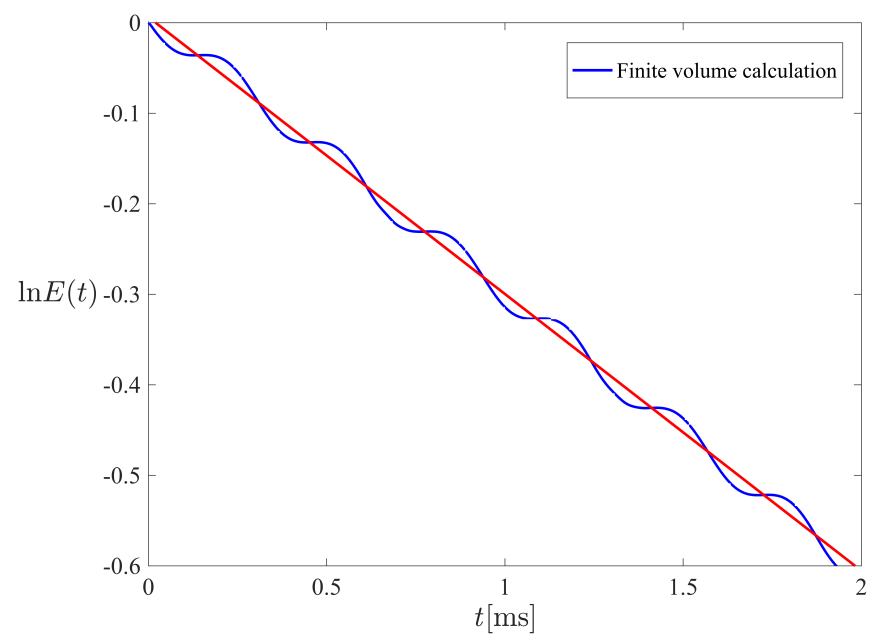

FIG. 4. Log of the mechanical energy stored in the PMMA slab (normalized by as function of $t(\mathrm{~ms})$, calculated using a finite-volume scheme (blue curve). The slope of its linear interpolation (red curve) matches $-2 \operatorname{Im} \omega$.

\section{THEORY FOR REAL PERTURBATIONS IN NON-HERMITIAN ELASTODYNAMICS: THE 1D MODEL PROBLEM}

In this last part, we are interested in developing a perturbation theory to the latter problem, noting that the obstacle lies in the divergence of $\Psi(x)$ at $\pm \infty$. Therefore, the orthogonality relations (27) no longer hold, and the components of $\mathrm{H}^{(0)}$ are unbounded when calculated according to Eq. (29). To overcome these obstacles, we first apply the complex scaling transformation $(x-l) \rightarrow(x-l) e^{i \theta}$ for $\Psi(x>l)$, with sufficiently large and real $\theta$ [52]. In these rotated coordinates, the transformed function

$$
\begin{aligned}
& \Psi_{n}(x>l ; \theta)=e^{i k_{b n}(x-l) e^{i\left(\theta-\varsigma_{n}\right)}} \\
& =e^{i\left|k_{b n}\right|(x-l) \cos \left(\theta-\varsigma_{n}\right)-\sin \left(\theta-\varsigma_{n}\right)} e^{-\left|k_{b n}\right|(x-l) \sin \left(\theta-\varsigma_{n}\right)}
\end{aligned}
$$

with $\tan \varsigma_{n}=-(n \pi)^{-1} \operatorname{arctanh} \gamma$, vanishes at infinity, owing to the second decaying exponent. Similarly, $\Psi(x<-l ; \theta)$ vanishes at $-\infty$ by applying the transformation $(x+l) \rightarrow$ $(x+l) e^{i \theta}$.

To establish next an orthonormal basis set, we replace the scalar product of the Hermitian formalism with the NHQM c-product [35], namely,

$\left(\Psi_{n}, \Psi_{n}\right):=\int_{-\infty}^{-l} \Psi_{n}^{2}(x ; \theta) \mathrm{d} x+\int_{-l}^{l} \Psi_{n}^{2}(x) \mathrm{d} x+\int_{l}^{\infty} \Psi_{n}^{2}(x ; \theta) \mathrm{d} x$,
To show that this product indeed delivers such a set, consider first the third term in Eq. (46). Since the scaled function vanishes at infinity, the integral is zero at its upper limit, and we are left with its value at $x=l$, such that

$$
\int_{l}^{\infty} \rho_{b} C_{n+}^{2} e^{2 i k_{b n}(x-l) e^{i \theta}} e^{i \theta} \mathrm{d} x=\frac{i \rho_{b}}{4 k_{b n}}\left(1 \pm \cos 2 k_{a n} l\right)
$$

where we used that fact that $C_{n+}=\cos k_{a n} l$ for the even modes (with a plus sign inside the brackets), and $C_{n+}=\sin k_{a n} l$ for the odd modes (minus sign). Owing to symmetry, this is also the value of the first integral in Eq. (46), and the remaining integral amounts to

$$
\int_{-l}^{l} \Psi_{n}^{2}(x) \mathrm{d} x=\rho_{a}\left(l \pm \frac{\sin 2 k_{a n} l}{2 k_{a n}}\right),
$$

where the plus and minus signs correspond to even and odd modes, respectively. We can now redefine the basis (28) to

$$
\hat{\Psi}_{n}^{(0)}=\Psi_{n}^{(0)} /\left(\Psi_{n}, \Psi_{n}\right),
$$

and observe that

$$
\left(\Psi_{n}(x), \Psi_{m}(x)\right)=\delta_{n m},
$$

when invoking Eq. (43).

As in Section IV, we replace again a part of the middle slab by a third constituent whose shear stiffness is $\alpha$; now, however, we leave the location, say $d$, and length, say $D-$ $d$, of the replacement as parameters. Using the framework developed in this Section, we can derive the eigenstates of the perturbed Hamiltonian as an expansion about a non-Hermitian Hamiltonian with real parameters. Specifically, we have that

$$
\mathrm{H}(\alpha, d, D)=\mathrm{H}^{(0)}+\alpha \mathrm{H}^{(1)}(d, D),
$$

where $\mathrm{H}^{(0)}$ is diagonal with complex eigenvalues associated with Eq. (43), and

$$
\mathrm{H}_{n m}^{(1)}=\int_{D}^{d} \Psi_{n}(x) \frac{\mathrm{d}^{2}}{\mathrm{~d} x^{2}} \Psi_{m}(x) \mathrm{d} x=k_{a m}^{2} \rho_{a} \int_{d}^{D} \Psi_{n}(x) \Psi_{m}(x) \mathrm{d} x,
$$

is a complex asymmetric matrix $\hat{\mathrm{H}}^{(1)}$ that depends nonlinearly on $d$ and $D$. (The resultant closed-form expressions are omitted here, for brevity). Notably, our developments further establish a platform for constructing degeneracies by real perturbations.

\section{SUMMARY AND OUTLOOK}

Motivated by the development of non-Hermitian quantum mechanics and the transfer of concepts from quantum theories to the macroscopic scale, we here revisit the connection between the time-independent Schrödinger equation and the one-dimensional elastodynamics equation. Using a simple transformation, we have first identified the term that appears in the elastodynamics equation and absent from the quantum 
mechanics equation. This derivation allowed us to determine the condition under which the two equations are equivalent.

Subsequently, we have showed the physical interpretation and application of different tools from non-Hermitian quantum mechanics in elastodynamics, including the timeindependent Rayleigh-Schrödinger perturbation theory to calculate the dynamic response of a finite elastic assembly; the non-Hermitian formalism of this theory to determine the perturbation series radius of convergence and exceptional point in the spectrum of the assembly; calculation of leaky modes and energy decay in an open elastic assembly using the poles of the scattering matrix; and the complex scaling transformation for establishing a basis from the corresponding divergent eigenfunctions.

Importantly, we have introduced a framework to analyze and design non-Hermitian degeneracies by real perturbations. These degeneracies have great potential in applications such as ultra-sensitive sensors and unidirectional energy scatterers, for which our approach offers a way to access without the need for gain or $\mathscr{P} \mathscr{T}$ symmetry. As a concrete application, we have demonstrated how an elastic slab assembly can function as enhanced mass sensor, when designed according to our analysis to exhibit two degenerate shear states. Using al- gebraic arguments and numerical calculations, we showed in Section V how the mass responsivity of this sensor surpasses the responsivity of conventional mechanical sensors owing to the square-root topology near the exceptional point in its spectrum.

We expect that our simplified demonstration of the potential that the tools non-Hermitian quantum mechanics has in elastodynamics will pave the way for further developments in more complex, practical elastic systems. Examples include periodic composites and homogenization [53-58], anisotropic media [59, 60], and elastodynamics in higher dimensions [61].

\section{ACKNOWLEDGMENTS}

This research was supported in parts by the I-Core: the Israeli Excellence Center "Circle of Light", the Israel Science Foundation (grants No. 1530/15 and 1912/15), the United States-Israel Binational Science Foundation (grant No. 2014358), and the ministry of science and technology. We thank Ron Ziv for sharing his MatLab code, and anonymous reviewers for constructive comments that helped us improve this paper.
[1] C Truesdell and W Noll. The Non-Linear Field Theories of Mechanics. Springer Science Business Media, Inc., 2004.

[2] Guancong Ma, Meng Xiao, and C. T. Chan. Topological phases in acoustic and mechanical systems. Nature Reviews Physics, 1(4):281-294, 2019.

[3] M. Miniaci, R. K. Pal, R. Manna, and M. Ruzzene. Valleybased splitting of topologically protected helical waves in elastic plates. Phys. Rev. B, 100:024304, Jul 2019.

[4] Natalia Lera, Daniel Torrent, P. San-Jose, J. Christensen, and J. V. Alvarez. Valley hall phases in kagome lattices. Phys. Rev. B, 99:134102, Apr 2019.

[5] Meng Xiao, Guancong Ma, Zhiyu Yang, Ping Sheng, Z. Q. Zhang, and C. T. Chan. Geometric phase and band inversion in periodic acoustic systems. NATURE PHYSICS, 11(3):240244, MAR 2015.

[6] A Srivastava. Metamaterial properties of periodic laminates. $J$. Mech. Phys. Solids, 96:252-263, 2016.

[7] J R Willis. Negative refraction in a laminate. J. Mech. Phys. Solids, 97:10-18, 2015.

[8] Sia Nemat-Nasser. Inherent negative refraction on acoustic branch of two dimensional phononic crystals. Mechanics of Materials, 132:1 - 8, 2019.

[9] Zhilin Hou, Huiqin Ni, and Badreddine Assouar. Pt-symmetry for elastic negative refraction. Phys. Rev. Applied, 10(4):44071, 2018.

[10] Ben Lustig, Guy Elbaz, Alan Muhafra, and Gal Shmuel. Anomalous energy transport in laminates with exceptional points. Journal of the Mechanics and Physics of Solids, page 103719, 2019.

[11] Christian E Rüter, Konstantinos G Makris, Ramy El-Ganainy, Demetrios N Christodoulides, Mordechai Segev, and Detlef Kip. Observation of parity-time symmetry in optics. Nature physics, 6(3):192, 2010.

[12] Eva-Maria Graefe and H. F. Jones. $\mathscr{P} \mathscr{T}$-symmetric sinusoidal optical lattices at the symmetry-breaking threshold. Phys. Rev. A, 84:013818, Jul 2011.

[13] Romain Fleury, Dimitrios Sounas, and Andrea Alù. An invisible acoustic sensor based on parity-time symmetry. Nature Communications, 6:5905, 2015.

[14] Steven A Cummer, Johan Christensen, and Andrea Alù. Controlling sound with acoustic metamaterials, 2016.

[15] J Christensen, M Willatzen, V R Velasco, and M.-H. Lu. Parity-Time Synthetic Phononic Media. Phys. Rev. Lett., 116(20):207601, 2016.

[16] V. Achilleos, G. Theocharis, O. Richoux, and V. Pagneux. Nonhermitian acoustic metamaterials: Role of exceptional points in sound absorption. Phys. Rev. B, 95:144303, Apr 2017.

[17] Zhilin Hou and Badreddine Assouar. Tunable elastic paritytime symmetric structure based on the shunted piezoelectric materials. Journal of Applied Physics, 123(8):85101, 2018.

[18] Aurélien Merkel, Vicent Romero-García, Jean-Philippe Groby, Jensen Li, and Johan Christensen. Unidirectional zero sonic reflection in passive $\mathscr{P} \mathscr{T}$-symmetric willis media. Phys. Rev. $B$, 98:201102, Nov 2018 .

[19] Carl M Bender and Stefan Boettcher. Real Spectra in NonHermitian Hamiltonians Having PT Symmetry. Phys. Rev. Lett., 80(24):5243-5246, 1998.

[20] Carl M Bender, Dorje C Brody, and Hugh F Jones. Complex Extension of Quantum Mechanics. Phys. Rev. Lett., 89(27):270401, 2002.

[21] Yan Lu and Ankit Srivastava. Level repulsion and band sorting in phononic crystals. Journal of the Mechanics and Physics of Solids, 111:100-112, 2018.

[22] Pu Zhang. Symmetry and degeneracy of phonon modes for periodic structures with glide symmetry. Journal of the Mechanics and Physics of Solids, 122:244 - 261, 2019.

[23] Joseph O. Hirschfelder, W. Byers Brown, and Saul T. Epstein. Recent developments in perturbation theory. volume 1 of $A d$ - 
vances in Quantum Chemistry, pages 255 - 374. Academic Press, 1964.

[24] F.M. Fernandez. Introduction to Perturbation Theory in Quantum Mechanics. CRC Press, 2000.

[25] Phillip R. Certain and Robert R. Merkel. Perturbation theory for short-range atomic interactions. ii. the radius of convergence. The Journal of Chemical Physics, 62(6):2406-2410, 1975.

[26] Nimrod Moiseyev and Shmuel Friedland. Association of resonance states with the incomplete spectrum of finite complexscaled Hamiltonian matrices. Phys. Rev. A, 22(2):618-624, 1980.

[27] Mohammad-Ali Miri and Andrea Alù. Exceptional points in optics and photonics. Science, 363(6422), 2019.

[28] Ş. K. Özdemir, S. Rotter, F. Nori, and L. Yang. Parity-time symmetry and exceptional points in photonics. Nature Materials, 18(8):783-798, 2019.

[29] Vincent Laude, Jose Maria Escalante, and Alejandro Martínez. Effect of loss on the dispersion relation of photonic and phononic crystals. Phys. Rev. B, 88:224302, Dec 2013.

[30] Fenfei Liu, Seyedhamidreza Alaie, Zayd C. Leseman, and Mani Hossein-Zadeh. Sub-pg mass sensing and measurement with an optomechanical oscillator. Optics Express, 21(17):1955519567, 2013.

[31] Yong He. Sensitivity of optical mass sensor enhanced by optomechanical coupling. Applied Physics Letters, 106(12):121905, 2019/12/12 2015.

[32] Jan Wiersig. Enhancing the sensitivity of frequency and energy splitting detection by using exceptional points: Application to microcavity sensors for single-particle detection. Phys. Rev. Lett., 112:203901, May 2014.

[33] Jan Wiersig. Sensors operating at exceptional points: General theory. Phys. Rev. A, 93:033809, Mar 2016.

[34] P. Djorwe, Y. Pennec, and B. Djafari-Rouhani. Exceptional point enhances sensitivity of optomechanical mass sensors. Phys. Rev. Applied, 12:024002, Aug 2019.

[35] Nimrod Moiseyev. Non-Hermitian Quantum Mechanics. Cambridge University Press, 2011.

[36] N. Moiseyev and P.R. Certain. Perturbation approach to the complex-rotation method. Molecular Physics, 37(5):16211632, 1979.

[37] Nimrod Moiseyev. Quantum theory of resonances: calculating energies, widths and cross-sections by complex scaling. Physics Reports, 302(5):212 - 293, 1998.

[38] Such a study will presumably require two-dimensional systems [10].

[39] Q. Zhong, J. Ren, M. Khajavikhan, D. N. Christodoulides, Ş. K. Özdemir, and R. El-Ganainy. Sensing with exceptional surfaces in order to combine sensitivity with robustness. Phys. Rev. Lett., 122:153902, Apr 2019.

[40] AA Mailybaev, ON Kirillov, and AP Seyranian. Geometric phase around exceptional points. PHYSICAL REVIEW A, 72(1), JUL 2005.

[41] Chen Shen, Junfei Li, Xiuyuan Peng, and Steven A. Cummer. Synthetic exceptional points and unidirectional zero reflection in non-hermitian acoustic systems. Phys. Rev. Materi- als, 2:125203, Dec 2018.

[42] Ramathasan Thevamaran, Richard Massey Branscomb, Eleana Makri, Paul Anzel, Demetrios Christodoulides, Tsampikos Kottos, and Edwin L. Thomas. Asymmetric acoustic energy transport in non-hermitian metamaterials. The Journal of the Acoustical Society of America, 146(1):863-872, 2019.

[43] K F Graff. Wave Motion in Elastic Solids. Dover Books on Physics Series. Dover Publications, 1975.

[44] Adi Pick and Nimrod Moiseyev. Polarization dependence of the propagation constant of leaky guided modes. Phys. Rev. A, 97:043854, Apr 2018.

[45] C. Cohen-Tannoudji, B. Diu, and F. Laloe. Quantum Mechanics. Number v. 1 in Quantum Mechanics. Wiley, 1991.

[46] Alexei A. Mailybaev. Computation of multiple eigenvalues and generalized eigenvectors for matrices dependent on parameters. Numerical Linear Algebra with Applications, 13(5):419-436, 2006.

[47] For asymmetric matrices, the notion of orthogonality is replaced with bi-orthogonality of right and left eigenvectors [35].

[48] Anja Boisen, Søren Dohn, Stephan Sylvest Keller, Silvan Schmid, and Maria Tenje. Cantilever-like micromechanical sensors. 74(3):036101, 2011.

[49] K. L. Ekinci, X. M. H. Huang, and M. L. Roukes. Ultrasensitive nanoelectromechanical mass detection. Applied Physics Letters, 84(22):4469-4471, 2019/12/12 2004.

[50] This choice has been made for numerical convenience, and approximates the model $\delta \rho(x)=m \delta(x)$, where $\delta(x)$ is the Dirac delta, such that its integral over $x$ equals $m$.

[51] Ron Ziv and Gal Shmuel. Observation of vector solitary waves in soft laminates using designated finite volume method. submitted, 2019.

[52] See Chapt. 5 in Ref. [35] and the references therein.

[53] Graeme W Milton. The theory of composites, volume 6. Cambridge university press, 2002.

[54] T Antonakakis, R V Craster, and S Guenneau. Homogenisation for elastic photonic crystals and dynamic anisotropy. J. Mech. Phys. Solids, 71:84-96, 2014.

[55] D Torrent, Y Pennec, and B Djafari-Rouhani. Effective medium theory for elastic metamaterials in thin elastic plates. Phys. Rev. $B, 90(10): 104110,2014$.

[56] G Shmuel and R Band. Universality of the frequency spectrum of laminates. J. Mech. Phys. Solids, 92:127-136, 2016.

[57] Ben Lustig and Gal Shmuel. On the band gap universality of multiphase laminates and its applications. Journal of the Mechanics and Physics of Solids, 117:37-53, 2018.

[58] Amir Ashkan Mokhtari, Yan Lu, and Ankit Srivastava. On the properties of phononic eigenvalue problems. Journal of the Mechanics and Physics of Solids, 2019.

[59] A N Stroh. Steady State Problems in Anisotropic Elasticity. Journal of Mathematics and Physics, 41(1-4):77-103, 1962.

[60] T C T Ting. Anisotropic Elasticity: Theory and Applications. Oxford Engineering Science Series. Oxford University Press, 1996.

[61] P G Ciarlet. Mathematical Elasticity, Volume I: ThreeDimensional Elasticity, Studies in Mathematics and its Applications. Elsevier, 1988. 